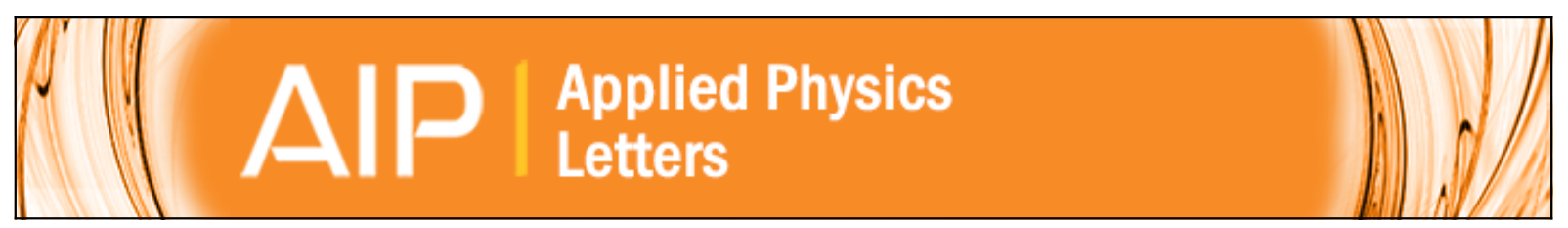

\title{
Simplistic graphene transfer process and its impact on contact resistance
}

Mohamed T. Ghoneim, Casey E. Smith, and Muhammad M. Hussain

Citation: Applied Physics Letters 102, 183115 (2013); doi: 10.1063/1.4804642

View online: http://dx.doi.org/10.1063/1.4804642

View Table of Contents: http://scitation.aip.org/content/aip/journal/apl/102/18?ver=pdfcov

Published by the AIP Publishing

\section{Articles you may be interested in}

Ferromagnetic tunnel contacts to graphene: Contact resistance and spin signal

J. Appl. Phys. 117, 083909 (2015); 10.1063/1.4913710

Reduction of metal contact resistance of graphene devices via CO2 cluster cleaning

Appl. Phys. Lett. 104, 223110 (2014); 10.1063/1.4881635

Clean transfer of graphene and its effect on contact resistance

Appl. Phys. Lett. 103, 103104 (2013); 10.1063/1.4819740

Thermal reversibility in electrical characteristics of ultraviolet/ozone-treated graphene

Appl. Phys. Lett. 103, 063107 (2013); 10.1063/1.4818329

Ultraviolet/ozone treatment to reduce metal-graphene contact resistance

Appl. Phys. Lett. 102, 183110 (2013); 10.1063/1.4804643

You don't

still use this

cell phone

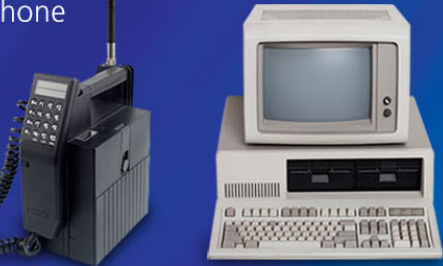

Why are you still using an AFM designed in the 80 's?
It is time to upgrade your AFM

Minimum $\$ 20,000$ trade-in discount for purchases before August 31st

Asylum Research is today's technology leader in AFM 


\title{
Simplistic graphene transfer process and its impact on contact resistance
}

\author{
Mohamed T. Ghoneim, Casey E. Smith, and Muhammad M. Hussain ${ }^{\text {a) }}$ \\ Integrated Nanotechnology Lab, Electrical Engineering, King Abdullah University of Science and Technology, \\ Thuwal 23955-6900, Saudi Arabia
}

(Received 21 February 2013; accepted 26 April 2013; published online 9 May 2013)

\begin{abstract}
Chemical vapor deposition based graphene grown on copper foil is attractive for electronic applications owing to its reliable growth process, large area coverage, and relatively defect free nature. However, transfer of the synthesized graphene to host substrate for subsequent device fabrication is extremely sensitive and can impact ultimate performance. Although ultra-high mobility is graphene's most prominent feature, problems with high contact resistance have severely limited its true potential. Therefore, we report a simple poly-(methyl methacrylate) based transfer process without post-annealing to achieve specific contact resistivity of $3.8 \times 10^{-5} \Omega \mathrm{cm}^{2}$ which shows $80 \%$ reduction compared to previously reported values. (C) 2013 AIP Publishing LLC. [http://dx.doi.org/10.1063/1.4804642]
\end{abstract}

Graphene has attracted much attention from the scientific community since its discovery in $2004 .^{1}$ Its ultra-high mobility and electrons behaving as massless fermions make it a strong candidate for high frequency electronic applications. $^{2,3}$ To date, a mobility of $200000 \mathrm{~cm}^{2} / \mathrm{V} \mathrm{s}$ has been reported for suspended graphene. ${ }^{4}$ Mechanical exfoliation, high temperature epitaxy, chemical synthesis, and chemical vapor deposition (CVD) based growth are the common methods to obtain graphene. ${ }^{5-11}$ Among these methods, CVD based growth on metal catalyst is considered the most economical pathway for synthesizing large area high quality graphene. Transfer of the graphene from metal catalyst to alternate substrates has been accomplished via exfoliation, Polydimethylsiloxane (PDMS) stamping, wafer bonding, etc. In the case of poly-(methyl methacrylate) (PMMA) assisted transfer, it is highly desirable to minimize the number of required cleaning/etching steps since physisorbed water and solvents as well as chemisorbed polymers and ion are known to affect graphene electronic properties. Specifically, many groups have reported complex transfer processes that disturb long range order in graphene, damage the graphene by mechanical wear and tear, and introduce dopants or sources of trapped charge. The dominant impact of these unintentional transfer related defects is high contact resistance due to poor metal/graphene interface. ${ }^{12}$ In the past, Robinson et al. reported specific contact resistivity of $2 \times 10^{-4} \Omega \mathrm{cm}^{2}$ for untreated (without any post processing) silicon carbide ( $\mathrm{SiC}$ ) based epitaxial graphene using titanium $(\mathrm{Ti}) /$ gold $(\mathrm{Au})$ metal bilayer. ${ }^{13}$ Next, Hsu et al. reported a modified method for achieving a contact resistance of 20-50 $\Omega$ after adding aluminum (Al) sacrificial layer, using AZ 5214 positive photoresist with image reversal capability and a tri-metal contact of titanium $(\mathrm{Ti}) /$ palladium $(\mathrm{Pd}) /$ gold $(\mathrm{Au})$, including expensive Pd. ${ }^{14}$ In this work, we report a contact resistance value of 27 $\Omega$ from atmospheric pressure chemical vapor deposition (APCVD) based graphene grown on copper $(\mathrm{Cu})$ foil, via a simpler route using ECI 3027 positive photoresist and without any post-processing. We also report a specific contact

\footnotetext{
${ }^{\text {a) }}$ Author to whom correspondence should be addressed. Electronic mail: muhammadmustafa.hussain@kaust.edu.sa
}

resistivity that is $80 \%$ lower than the value reported by Robinson et al. ${ }^{13}$

CVD graphene was grown at atmospheric pressure on $\mathrm{Cu}$ foil using methane and hydrogen at $1000^{\circ} \mathrm{C}$. The growth phase was terminated by nitrogen purge and cooling under ambient conditions. Figure 1(a) shows the intensity ratio of the $2 \mathrm{D} / \mathrm{G}$ peaks of our as grown graphene. The mapping result shows that the ratio ranges predominantly between 0.45 and 0.65 indicating few layers of graphene (FLG). Although it is desirable to minimize thickness variation for controllable wafer scale production, it was experimentally reported that the number of layers has no effect on contact resistance measurements. ${ }^{15}$ Figure 1(b) shows that graphene showed fairly low defectivity with an $\mathrm{I}_{\mathrm{G}} / \mathrm{I}_{\mathrm{D}}$ ratio of $\sim 4.5$ on average. It is expected that further optimization of synthesized graphene defectivity would yield better quality metal/ graphene interfaces and consequently lower contact resistance. After preliminary characterization of graphene sheets, polymer supported transfer was carried out to position synthesized graphene on a silicon wafer with $300 \mathrm{~nm}$ plasma enhanced chemical vapor deposition (PECVD) based silicon dioxide isolation layer. The transfer process is described in Figure 2. Figure 3(a) shows a high resolution optical image for the transferred graphene sheet (inset shows the graphene flake on silicon oxide). It is evident from the microscopic visual inspection that the graphene sheet lacks severe cracks or folds caused during mechanical handling. Atomic force microscopy (AFM) was conducted on a $20 \times 20 \mu \mathrm{m}^{2}$ section of the transferred film and showed an $\mathrm{R}_{\mathrm{rms}}$ surface roughness value of $15.7 \mathrm{~nm}$ [Figure 3(b)]. The surface roughness value is higher than expected for our FLG and can be attributed to trapped water molecules and hydrocarbons adsorbed from ambient between the graphene sheet and the $\mathrm{SiO}_{2}$ substrate. Since extensive studies have been done in the past on the effect of surface treatment of the $\mathrm{SiO}_{2}$ layer on the quality of the graphene/ $\mathrm{SiO}_{2}$ interface and consequently mobility, ${ }^{16}$ in this study we limit our study to optimizing the metal/graphene interface under the assumption that graphene/ $\mathrm{SiO}_{2}$ interface optimization research can be applied to build on and further improve our results. Finally, transfer length method (TLM) was used to collect contact resistance and 


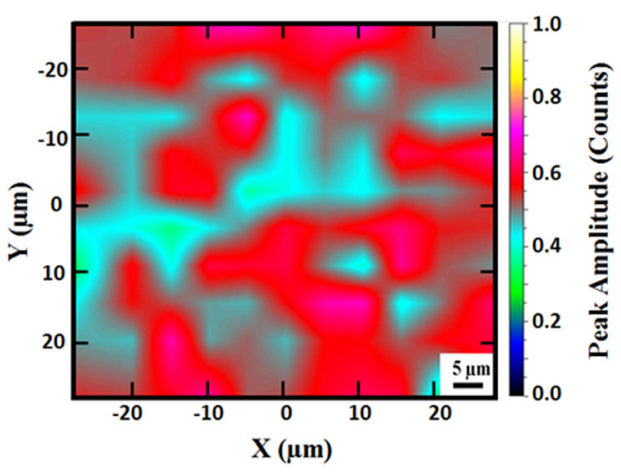

(a)

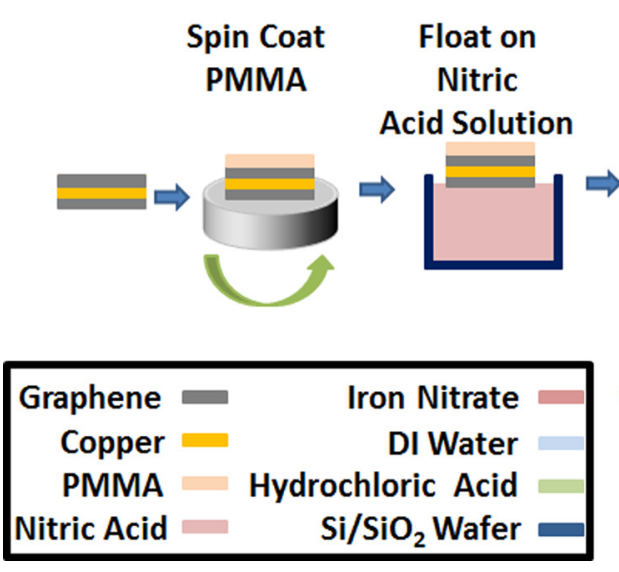

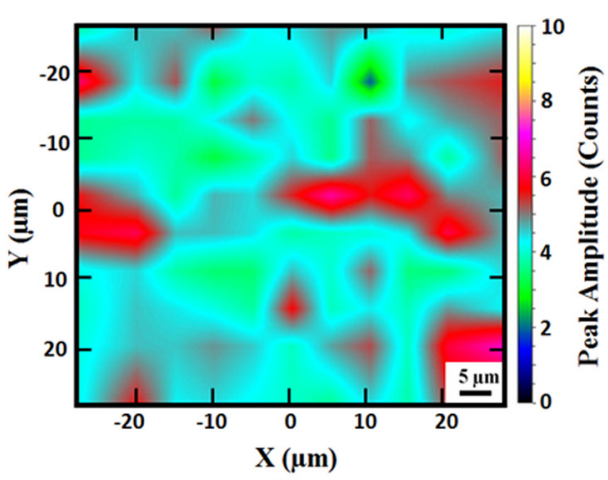

(b)

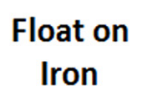
Iron Nitrate

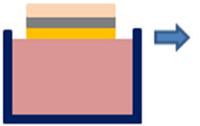

Float on Hydrochloric Acid Rinse in Acetone
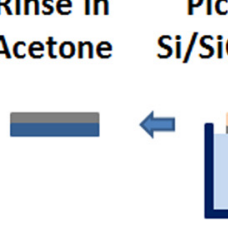

specific contact resistivity measurements. Bi-layer metal contacts of $10 \mathrm{~nm}$ titanium $(\mathrm{Ti}) / 50 \mathrm{~nm}$ gold $(\mathrm{Au})$ were deposited using thermal e-beam evaporation without breaking the vacuum to avoid the formation of any metal oxides at the Ti/Au interface. The contacts were patterned using a lift-off process utilizing $4 \mu \mathrm{m}$ of ECI 3027 positive photoresist. No posttransfer processing steps after PMMA removal were undertaken. TLM measurements were collected using 2-probes configuration on non-isolated graphene structures with $200 \times 200 \mu \mathrm{m}^{2}$ contacts skipping the $\mathrm{O}_{2}$ plasma etching of graphene. This approach was based on the assumption that since current injection takes place at the edge of the metal/graphene contact, ${ }^{17}$ electrons will preferentially constrain their path from source to drain through a channel of width equal to the contact metal edge. To ensure this has no effect on our contact resistance measurements, we did a control experiment with non-isolated graphene and isolated/patterned graphene structures and found that the contact resistance values did not

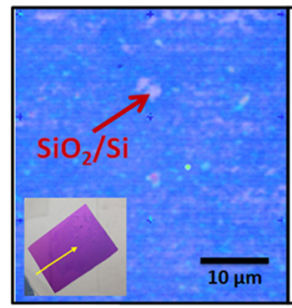

(a)

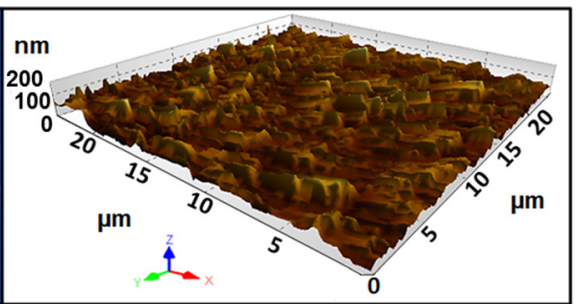

(b)

FIG. 3. (a) High resolution optical image of PMMA transferred graphene sheet (inset shows the graphene flake on $\mathrm{SiO}_{2}$ ) and (b) AFM profile for $20 \times 20 \mu \mathrm{m}^{2}$ transferred sample area.

FIG. 1. Raman mapping of (a) $2 \mathrm{D} / \mathrm{G}$ intensity ratio of as grown graphene for a $50 \times 50 \mu \mathrm{m}^{2}$ area showing predominantly a ratio between 0.45 and 0.65 indicating FLG and (b) G/D intensity ratio of as grown graphene showing a 4.5 ratio on average indicating acceptable quality.

FIG. 2. Polymer supported transfer of graphene using PMMA. First, top side graphene is coated with PMMA, followed by back side graphene etch in nitric acid $\left(\mathrm{HNO}_{3}\right)$. Then copper $(\mathrm{Cu})$ is etched in iron nitrate $\left(\mathrm{FeNO}_{3}\right)$ solution followed by deionized (DI) water. Hydrochloric acid $(\mathrm{HCl})$ is used afterwards to wash away copper etchant residues followed by another DI water bath. Finally, scooping with target substrate is done. change (see supporting information). ${ }^{18}$ Then we swept the voltage from -1 to $1 \mathrm{~V}$ to deduce the resistance values.

Figure 4 shows the best fit for the TLM measurements collected. The $\mathrm{R}^{2}$ (coefficient of determination (COD)) for the plot is 0.99 indicating that the constructed model accurately represents data. Every point on the plot is a resultant of taking the median from 7 measurements for contacts separated by the same distance from different devices on the same substrate to minimize process variations effect. From this exercise, we extract a specific contact resistivity value of $3.8 \times 10^{-5} \Omega \mathrm{cm}^{2}$ which is a 5 folds reduction in specific contact resistivity than previously reported for no-post

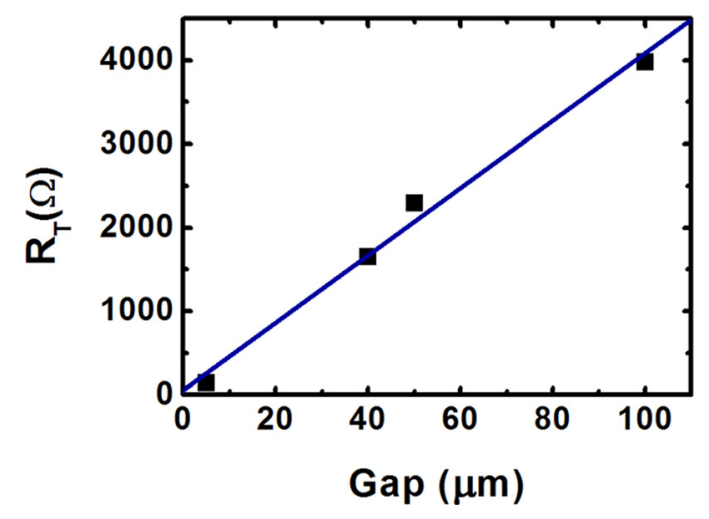

FIG. 4. Contact resistance plot for best fit using TLM for PMMA transferred graphene. Every point shown represents the median of 7 resistance measurements separated by the same distance and the $\mathrm{R}^{2}$ (COD) is 0.99 which adds to the accuracy of the data and compensates for having more points. The COD value indicates the accuracy of the best fit line with respect to data. The closer to " 1 " the COD, the more accurate the fit is and the more representative of data points. 
TABLE I. Comparison of various published literature data on contact resistances or specific contact resistivity from various metal/graphene contacts.

\begin{tabular}{|c|c|c|c|c|c|}
\hline Refs. & Growth method & Transfer method & Contact metal & Contact resistance $(\Omega)$ & Specific contact resistivity $\left(\Omega \mathrm{cm}^{2}\right)$ \\
\hline 15 & Exfoliation & $\ldots$ & $\mathrm{Ni}$ & $490-1090$ & $\ldots$ \\
\hline 20 & Graphene oxide reduction & $\ldots$ & $\mathrm{W}$ & $\ldots$ & $1 \times 10^{-6}$ \\
\hline 21 & Graphene oxide reduction & $\ldots$ & $\mathrm{Al}, \mathrm{Cr}, \mathrm{Pd}$, and $\mathrm{Yb}$ & $\ldots$ & $\ldots$ \\
\hline 17 & Exfoliation & $\ldots$ & $\mathrm{Ni}$ & $\ldots$ & $5 \times 10^{-6}$ \\
\hline 22 & Exfoliation & $\ldots$ & $\mathrm{Ti} / \mathrm{Au}$ & 45.5 & $\ldots$ \\
\hline 14 & $\mathrm{CVD}$ on $\mathrm{Cu}$ & PMMA transfer & $\mathrm{Ti} / \mathrm{Pd} / \mathrm{Au}$ & 20 & $\ldots$ \\
\hline 13 & $\mathrm{SiC}$ & $\ldots$ & $\mathrm{Ti} / \mathrm{Au}$ & $\ldots$ & $2 \times 10^{-4}$ \\
\hline 23 & CVD on $\mathrm{Ni}$ & PMMA transfer & $\mathrm{Ti} / \mathrm{Pd} / \mathrm{Au}$ & $\ldots$ & $2 \times 10^{-6}$ \\
\hline 24 & $\mathrm{SiC}$ & $\ldots$ & $\mathrm{Cr} / \mathrm{Au}$ & $\ldots$ & $5 \times 10^{-3}$ \\
\hline 25 & $\mathrm{CVD}$ on $\mathrm{Cu}$ & PMMA transfer & $\mathrm{Pd}$ & $\ldots$ & $\ldots$ \\
\hline 26 & $\mathrm{CVD}$ on $\mathrm{Cu}$ & PMMA transfer & $\mathrm{Au}$ & 340 & $\ldots$ \\
\hline This work & $\mathrm{CVD}$ on $\mathrm{Cu}$ & PMMA transfer & $\mathrm{Ti} / \mathrm{Au}$ & 27 & $3.8 \times 10^{-5}$ \\
\hline
\end{tabular}

processed graphene epitaxially grown on $\mathrm{SiC}$. This is a significant step towards industrialization of graphene processes as $\mathrm{SiC}$ epitaxially grown graphene is limited by the size and cost of SiC wafers. Hence, obtaining comparable results using CVD grown graphene with no post-transfer processing has a higher potential for device application due to its lower specific contact resistivity added to the cost and scalability advantage of CVD graphene over $\mathrm{SiC}$ epitaxially grown graphene. $^{19}$

Table I provides an overview of the graphene contact resistance research over the past two years. Most notable is the experiment of Allen Hsu et al. ${ }^{14}$ In that work, CVD grown graphene was also transferred using PMMA before contacting the graphene. Lift-off processing with image reversed AZ 5214E photoresist $(\sim 1.5 \mu \mathrm{m}$ thickness) requires additional reversal bake and flood exposure steps to generate a re-entrant resist profile. We compensated for the difficulty in creating a similar profile with positive resist by increasing the thickness ( $4 \mu \mathrm{m}$ of ECI 3027$)$ and intentionally underexposing to achieve effective lift-off eliminating the extra processing steps involved with AZ 5214E. We observe that additional bake and exposure steps required with AZ 5214E typically harden the resist against solvent based removal and could leave more residue at the metal/graphene interface. Another critical difference is that a sacrificial layer that is directly deposited and removed from the graphene sheet was not required to achieve low contact resistance. Although a $5 \mathrm{~nm}$ of aluminum (Al) sacrificial layer by e-beam evaporation on graphene was suggested to improve the surface roughness of the graphene sheet, ${ }^{14}$ our study suggests that the deposition and removal step degrade graphene such that the surface roughness improvement is counteracted. This is supported by eliminating the other possibility that surface roughness has no significant effect on contact resistance. Certainly as surface roughness increases, the quality of the metal/graphene decreases leading to higher contact resistance as proven experimentally by $\mathrm{Hsu}$ et al. ${ }^{14}$ Added to the damaging effects this might have on graphene, there are other issues specific to e-beam evaporation. It is difficult to control the thickness variation using e-beam evaporation and it has poor step coverage. ${ }^{27}$ Our study also uses a bi-layer contact $(\mathrm{Ti} / \mathrm{Au})$ instead of a tri-metal contact of $1.5 \mathrm{~nm} \mathrm{Ti} /$ $45 \mathrm{~nm} \mathrm{Pd} / 15 \mathrm{~nm} \mathrm{Au}$. It is to be noted that, due to the limited density of states in graphene near the Dirac point, ${ }^{12}$ the contact resistance is not limited by the work function of the metal but by the available empty states in graphene. Supporting Table I highlights the difference between the processes used in Al sacrificial contacts versus this work's process. ${ }^{18}$ Therefore, we have demonstrated a simpler route to comparable results via a more environmentally benign solution avoiding high temperature forming gas anneal, $\mathrm{Al}$ and expensive Pd e-beam evaporation, and extra baking steps, while preserving graphene sheet quality intact as that of an as-grown graphene sheet. Furthermore, it is important to highlight the economic advantage utilizing CVD grown graphene that produces wafer scale graphene sheets compared to the expensive epitaxially grown few microns graphene on SiC. In addition, compared to other demonstrations using exfoliated graphene or graphene reduced from graphite oxide, we stand out from the performance per process complexity perspective. It is to be noted that although Huang et al. used CVD graphene grown on nickel, they used back gated device to reduce specific contact resistivity significantly which can definitely reduce our specific contact resistivity further.

In summary, we have reported a simple route for PMMA based transfer of CVD grown graphene from copper foil. We have used a $\mathrm{Ti} / \mathrm{Au}$ contact deposited by e-beam evaporation without any vacuum break to achieve a contact resistivity of $3.8 \times 10^{-5} \Omega \mathrm{cm}^{2}$ which is $80 \%$ lower than the previously reported value obtained from epitaxially grown graphene. This result also shows the comparable contact resistance to earlier results achieved using tri-metal contacts and additional process steps. We attribute gentler processing performed during delicate graphene transfer process to reduce the surface damage and possible doping leading to attractive contact resistance.

This work was supported under Competitive Research Grant Funding Program (CRG-1-2012-HUS-008) by KAUST Office of Competitive Research Funds (OCRF).

${ }^{1}$ K. S. Novoselov, A. K. Geim, S. V. Morozov, D. Jiang, Y. Zhang, S. V. Dubonos, I. V. Grigorieva, and A. A. Firsov, Science 306(5696), 666 (2004).

${ }^{2}$ A. K. Geim and K. S. Novoselov, Nature Mater. 6, 183 (2007). 
${ }^{3}$ F. Schwierz, Nat. Nanotechnol. 5, 487 (2010).

${ }^{4}$ K. I. Bolotin, K. J. Sikes, Z. Jiang, M. Klima, G. Fudenberg, J. Hone, P. Kim, and H. L. Stormer, Solid State Commun. 146, 351 (2008).

${ }^{5}$ K. S. Novoselov, D. Jiang, F. Schedin, T. J. Booth, V. V. Khotkevich, S. V. Morozov, and A. K. Geim, Proc. Natl. Acad. Sci. U.S.A. 102(30), 10451 (2005).

${ }^{6}$ C. Berger, Z. Song, X. Li, X. Wu, N. Brown, C. Naud, D. Mayou, T. Li, J. Hass, A. N. Marchenkov, E. H. Conrad, P. N. First, and W. A. de Heer, Science 312, 1191 (2006).

${ }^{7}$ D. Li, M. B. Müller, S. Gilje, R. B. Kaner, and G. G. Wallace, Nat. Nanotechnol. 3(2), 101 (2008).

${ }^{8}$ X. Li, X. Wang, L. Zhang, S. Lee, and H. Dai, Science 319, 1229 (2008).

${ }^{9}$ D. V. Kosynkin, A. L. Higginbotham, A. Sinitskii, J. R. Lomeda, A. Dimiev, B. K. Price, and J. M. Tour, Nature 458, 872 (2009).

${ }^{10}$ X. Li, W. Cai, J. An, S. Kim, J. Nah, D. Yang, R. Piner, A. Velamakanni, I. Jung, E. Tutuc, S. K. Banerjee, L. Colombo, and R. S. Ruoff, Science 324(5932), 1312 (2009).

${ }^{11}$ A. Reina, X. Jia, J. Ho, D. Nezich, H. Son, V. Bulovic, M. S. Dresselhaus, and J. Kong, Nano Lett. 9(1), 30 (2009).

${ }^{12}$ K. Nagashio, T. Nishimura, K. Kita, and A. Toriumi, Tech. Dig. - Int. Electron Devices Meet. 2009, 1.

${ }^{13}$ J. A. Robinson, M. LaBella, M. Zhu, M. Hollander, R. Kasarda, Z. Hughes, K. Trumbull, R. Cavalero, and D. Snyder, Appl. Phys. Lett. 98, 053103 (2011).

${ }^{14}$ A. Hsu, H. Wang, K. K. Kim, J. Kong, and T. Palacios, IEEE Electron. Device Lett. 32(80), 1008 (2011).

${ }^{15}$ A. Venugopal, L. Colombo, and E. M. Vogel, Appl. Phys. Lett. 96, 013512 (2010).
${ }^{16}$ K. Nagashio, T. Yamashita, J. Fujita, T. Nishimura, K. Kita, and A. Toriumi, Tech. Dig. - Int. Electron Devices Meet. 2010, 23.4.1.

${ }^{17}$ K. Nagashio, T. Nishimura, K. Kita, and A. Toriumi, Appl. Phys. Lett. 97, 143514 (2010).

${ }^{18}$ See supplementary material at http://dx.doi.org/10.1063/1.4804642 for contact resistance measurement plot and method in tabular format and comparison of process steps in Ref. 14 and this work.

${ }^{19}$ D. R. Cooper, B. D'Anjou, N. Ghattamaneni, B. Harack, M. Hilke, A. Horth, N. Majlis, M. Massicotte, L. Vandsburger, E. Whiteway, and V. Yu, ISRN Condens. Matter Phys. 2012, 501686.

${ }^{20}$ F. P. Rouxino, R. V. Gelamo, R. G. Amici, A. R. Vaz, and S. A. Moshkalev, Appl. Phys. Lett. 97, 253104 (2010).

${ }^{21}$ A. Nourbakhsh, M. Cantoro, A. Hadipour, T. Vosch, M. H. van der Veen, M. M. Heyns, B. F. Sels, and S. De Gendt, Appl. Phys. Lett. 97, 163101 (2010).

${ }^{22}$ S. Russo, M. F. Craciun, M. Yamamoto, A. F. Morpurgo, and S. Tarucha, Physica E 42(4), 677 (2010).

${ }^{23}$ B.-C. Huang, M. Zhang, Y. Wang, and J. Woo, Appl. Phys. Lett. 99, 032107 (2011).

${ }^{24}$ V. K. Nagareddy, I. P. Nikitina, D. K. Gaskill, J. L. Tedesco, R. L. MyersWard, C. R. Eddy, J. P. Goss, N. G. Wright, and A. B. Horsfall, Appl. Phys. Lett. 99, 073506 (2011).

${ }^{25}$ M. S. Choi, S. H. Lee, and W. J. Yoo, J. Appl. Phys. 110, 073305 (2011).

${ }^{26}$ R. S. Sundaram, M. Steiner, H.-Y. Chiu, M. Engel, A. A. Bol, R. Krupke, M. Burghard, K. Kern, and P. Avouris, Nano Lett. 11(9), 3833 (2011).

${ }^{27}$ C. Y. Kang, R. Choi, M. M. Hussain, J. Wang, Y. J. Suh, H. C. Floresca, M. J. Kim, J. Kim, B. H. Lee, and R. Jammy, Appl. Phys. Lett. 91, 033511 (2007). 